\title{
La investigación sobre lectura en el entorno digital
}

\section{Research on reading in the digital environment}

\author{
José Antonio Cordón García \\ jcordon@usal.es \\ Catedrático de Bibliografía. Universidad de Salamanca.
}

\section{Resumen}

La investigación sobre la lectura durante décadas ha centrado su atención en los procesos de alfabetización, en la formación del lector, en las dificultades para el desarrollo de competencias lectoras y en los patrones de comportamiento de los lectores desde un punto de vista estadístico. La aparición de dispositivos de lectura electrónica, y de contenidos digitales sobre todo a finales del siglo XX, ha generado una corriente de investigación orientada a examinar las circunstancias en las que está produciendo la migración de un sistema analógico a otro digital y la incidencia de la tecnología en algunas de las funciones asociadas con la práctica lectora, como la concentración, la memorización y la interpretación. En este artículo se traza un panorama del desarrollo de algunas de estas investigaciones, estableciendo algunos de los elementos críticos para la consolidación de las mismas.

\section{Palabras clave}

Lectura, Lectura digital, dispositivos de lectura, Comprensión lectora.

\begin{abstract}
Research on reading has focused on literacy processes, reader training, difficulties in developing reading skills, and on the behavioural patterns of readers from a statistical point of view. The emergence of electronic reading devices and digital content, especially at the end of the 20th century, has generated a stream of research aimed at examining the circumstances in which the migration from an analogue to a digital system is taking place and the incidence of technology in some of the functions associated with reading practice, such as concentration, memorization and interpretation. This article presents an overview of the development of some of these investigations, establishing some of the critical elements for their consolidation.
\end{abstract}




\section{Keywords}

Reading, Digital reading, Electronic devices, Reading comprehension,

Recibido: 02/12/2016

Aceptado: 19/12/2016

DOI: http:/ / dx.doi.org/10.5557/IIMEI7-N13-247268

Descripción propuesta: CORDÓN GARCÍA, José Antonio, 2016. La investigación sobre lectura en el entorno digital. Métodos de Información [en línea], 7(13), pp. 247-268.

\section{https://creativecommons.org/licenses/by/4.0/}

\section{La investigación sobre el libro y la lectura en el contexto impreso}

Durante muchos años la investigación sobre el libro y la lectura se había centrado fundamentalmente en algunos de los elementos constitutivos del campo editorial, esto es los relacionados con la creación y la producción, y en algunos casos la recepción, pero esta última considerada en tanto que sistema de información sobre ventas y patrones de comportamiento del público lector-comprador. El lector y la lectura han recibido poca atención fuera de la conformación de estos patrones estadísticos y la investigación relacionada con la alfabetización, sus modelos, dificultades y patologías. Educación y Psicología concitan la mayoría de las investigaciones desarrolladas durante los últimos 20 años sobre el tema. La premisa subyacente en todas estas investigaciones es que la lectura constituye un bien en sí mismo y que la adquisición de las competencias para su desarrollo constituyen un factor inherente a la conformación de la persona, por lo que es preciso analizar las condiciones para su asimilación y resolver cualquier tipo de problema que se interponga para la consecución del mismo. El otro gran foco de los estudios sobre la lectura está representado por los análisis de carácter sociológico, efectuados a partir de las series estadísticas que, en casi todos los países, se viene efectuando desde los años 60 para examinar los hábitos de lectura de la población, siempre desde la premisa de que cualquier política cultural ha de estimular una forma particular de lectura, la de libros, que se erige en referente del bien leer. Desde esta perspectiva, el concepto de "campo" es una de las aportaciones más afortunadas de Pierre Bourdieu a los estudios sociológicos. 
Su traslación al mundo de la creación cultural en general y al sector de la literatura en particular, permitió definir una gran cantidad de conceptos y fenómenos cuya interrelación se hizo desde entonces evidente, articulando un modelo de análisis que ha gozado del respaldo y atención de numerosos investigadores. Bourdieu establece un conjunto de fuerzas cuyo desarrollo funciona como sistema de legitimación de los diferentes actores que intervienen en el sector editorial, erigiendo un juego de equilibrios, ponderaciones y desplazamientos, gracias a los cuales se produce la prevalencia y jerarquías con que son percibidos y reconocidos por sus pares. En algunas de sus obras (Bourdieu, 2011, 2012) aborda la situación del lector en relación con el resto de componentes del entorno de la creación cultural, otorgándole una posición que está en consonancia con las circunstancias de su formación, pertenencia de clase y horizonte de expectativas. El lector constituye uno de los componentes esenciales en el estudio de las interrelaciones generadas en el mundo editorial, un elemento interdependiente, como lo definiera Even Zohar (1994), en su teoría de los polisistemas, cuya condición está estrechamente relacionada con el conjunto de actores en juego.

En esta misma línea la teoría de la recepción promovió la investigación sobre el lector a un primer plano situándolo en una posición activa respecto al texto, con capacidad para conformarlo y adaptarlo según su bagaje y experiencias. En este sentido Jauss (2012), Iser (1987, 2005) Fish (1990) y Bleich (1978), entre otros, renovaron los estudios sobre la lectura, iniciando una corriente de investigación que invertía el polo de atención, bien hacia los horizontes de expectativas que generaba un texto (Jauss e Iser), bien hacia la representación de la misma que hacía el lector (Fish y Bleich).

La estética de la recepción plantea una revisión de la consideración tradicional del libro como objeto, para inscribirlo en un sistema de interpretaciones en las que prevalecen sus características sociales, intelectuales y organizativas. Se trata de un giro significativo que sitúa el foco en un conjunto de elementos de carácter más difuso y complejo que los relacionados con la investigación de las fisicidades vinculadas con el libro objeto (Houston, 2016), pero que van articulando un cuerpo de conocimientos cada vez más sólidos.

Hasta comienzos del siglo XXI la lectura, el lector y su estudio se había movido en un territorio cómodo sólidamente establecido, asentado en 
contextos de investigación de larga tradición provenientes, como se ha comentado más arriba, de los campos de la Psicología, la Educación, la Sociología y, subsidiariamente (en lo referente a los estudios sobre autoria e historia del libro) la Filología y la Historia. La aparición de la web, el hipertexto y diferentes tecnologías de la información y la comunicación, han modificado profundamente las perspectivas metodológicas desde las que se aborda la lectura en general, y la lectura digital en particular, introduciendo un conjunto de variables ergonómicas, perceptivas, cognoscitivas, emocionales, fenomenológicas, y socioculturales que vuelven ineludible la dimensión multidisciplinar de la investigación (Mangen; Van der Weel, 2016)

\section{E1 lector y la lectura en el entorno digital}

Las nociones de autor, editor, crítico, lector, libro, etc. revestían unas características muy precisas que era posible estudiar con metodologías claramente establecidas por las diferentes corrientes de análisis. Incluso los autores más innovadores, que comienzan a introducir elementos disruptivos en sus planteamientos, como Chartier (2000), Furtado (2007) o Darnton (2010), se movían en un terreno conocido, cuyo referente era el impreso, para dilucidar los nuevos entornos.

Pero el contexto en el que se desarrolla la lectura en la actualidad, aun respondiendo en gran medida, todavía, a los modelos heredados de siglos, cuenta, entre otros muchos, con un elemento inexistente previamente, el dispositivo de lectura, la pantalla, un factor determinante que rompe con una tradición centenaria y que introduce un factor disruptivo al que se le ha prestado escasa atención. Es cierto, como señala Chartier (2000), que nos encontramos inmersos en una revolución de las formas de producción, de los soportes y de las prácticas de lectura, pero lejos de atender a la misma, como un estadio evolutivo más, es preciso prestar atención, como acertadamente señalara el propio Chartier en conversación con Pierre Bourdieu, a las "discontinuidades" (Bourdieu; Chartier, 2011), a la aparición de hechos o circunstancias para los que la investigación ha de aportar nuevas herramientas de análisis y marcos de interpretación.

La aparición de un sistema de intermediación modifica radicalmente todo el ecosistema del libro, pues la relación del lector con la obra, la del editor con el 
lector, la del lector con el autor, etc, cambia radicalmente según los contextos y el modo en el que se desarrollan los contenidos. La mediación tecnológica introduce un elemento nuevo en el proceso lector, vinculado con la apropiación del dispositivo y con la ejecución del contenido. No basta con saber leer, sino que es preciso controlar donde se lee y, sobre todo, como se lee.

Aunque se publican muchos informes sobre la evolución de la publicación de libros electrónicos, o de la lectura digital, interesantes para comprobar la progresión que van experimentando ambos segmentos en el mundo, no contamos con suficientes investigaciones que indaguen en la naturaleza de cómo se está produciendo la transformación en las prácticas de lecto-escritura en la fase de transición en la que nos encontramos

¿Qué sabemos acerca de la forma en que interactuamos con los nuevos soportes que sustituyen a los medios convencionales? ¿Cómo se representa y gestiona el conocimiento y la experiencia del pasado a través de los nuevos medios? ¿Cuáles son las formas en que nos relacionamos con estos objetos de conocimiento y la información que contienen? ¿Qué impacto reviste la confluencia de formatos y medios en la experiencia de lectura? ¿Qué tipo de interacciones se producen entre los usuarios y los artefactos digitales?, son algunas de las preguntas a las que es preciso responder para articular una teoría sólida de la lectura digital.

Igual que la fiabilidad representada por la construcción social de la imprenta ha constituido una pregunta de investigación importante para los estudiosos de la historia del libro, la aceptación social de lo digital como sistema de verificación social representa un campo de estudio ineludible en nuestro ámbito. La construcción del lector digital, pero también la del editor o el autor digital. El estudio del público y de las audiencias implica el análisis de cómo se transforma una práctica analógica en digital, como se concilian ambas prácticas, o como se excluye una en beneficio de otra.

Para poder evaluar e interpretar correctamente las prestaciones y valores de los dispositivos de lectura y de los contenidos de los mismos, su aptitud para fines de investigación o de lectura convencional, es preciso conocer cuáles son los comportamientos de estos usuarios en entornos digitales ¿qué tipo de recursos necesitan, que recursos utilizan, por qué se produce el rechazo o aceptación de unos en detrimento de otros?. Uno de los objetivos de la 
investigación debe ser identificar y evaluar el impacto de las variables contextuales en la interacción con los textos electrónicos. Esto incluye el análisis de tareas y el desarrollo de taxonomías para los distintos tipos de interacción, los escenarios de uso y las funciones del usuario.

La modificación de los procesos de producción afecta de manera fundamental a las nociones culturales de autoría, lectores, y forma literaria. Por ejemplo, el elevado coste de los materiales necesarios para producir un libro impreso obligó a la publicación selectiva de acuerdo con unos criterios editoriales que devinieron en factores críticos del sistema de publicación: calidad de los contenidos, capacidad de venta del autor, circulación de la temática, etc. ¿Al cambiar la tecnología y el proceso se modifica el tipo de lectores? ¿Es decir, qué elementos de los libros impresos son valiosos o incluso imprescindibles en el nuevo contexto digital, y qué nuevas funciones son lo suficientemente importantes para lectores e investigadores y susceptibles de desarrollo?

Son pocos los programas de investigación que han abordado los elementos inherentes a la migración digital y a las prácticas generadas en los nuevos entornos. En España solo el programa Territorio Ebook, desarrollado por la Fundación Germán Sánchez Ruipérez, durante casi una década, ha abordado con solvencia el recorrido de la lectura impresa a la digital, articulando una etnografía del lector digital rigurosa y exhaustiva, contemplando todos los grupos de edad.

\section{Las interfaces de lectura y la triada manipulación, comprensión, interpretación}

Las aplicaciones de lectura se han convertido en importantes interfaces para la comprensión e interpretación de una obra, aportando elementos contextuales que facilitan su legibilidad y asimilación (Gómez et. al, 2016). Ahora bien, ¿Cómo se utilizan las herramientas y aplicaciones en la lectura o el estudio de un libro? ¿De qué manera se han incorporado en la sociedad en general o en la comunidad académica? ¿Cuáles son los resultados esperados en la lectura de un texto? ¿Qué tipo de herramientas facilitan o pueden facilitar la comprensión de un texto, y cómo aparecen estas funciones en los entornos de lectura? ¿Las nuevas herramientas de lectura están diseñadas para extender el uso de esta? ¿qué funciones están implícitas en la lectura? ¿Cuáles son las 
posibilidades de las distintas interfaces y como se aprovechan? ¿Qué nuevos tipos de interfaces están surgiendo?

Estas interrogantes alcanzan su máxima expresión cuando consideramos que la interfaz de lectura adquiere una importancia capital para el acceso al contenido, y que sus disposiciones funcionales, tanto en lo que al dispositivo se refiere como a las formas en que las aplicaciones condicionan los modos de leer (Emerson, 2014), representan sistemas de interposición entre el lector y el contenido, afectando, además a este.

Los textos impresos en papel presentan un conjunto de contingencias sensomotoras que difieren de los textos mostrados en un ordenador, tableta, o en un dispositivo de tinta electrónica. Los textos impresos son estables y tangibles; están unidos físicamente al soporte. Por el contrario, los textos en pantalla son intangibles y virtuales, están separados físicamente de su soporte. Esta estructura permite que los dispositivos digitales puedan almacenar y mostrar una serie de textos (documentos; libros) proporcionando una extraordinaria portabilidad y accesibilidad. Sin embargo, la propia flexibilidad puede alterar la relación del lector con el texto, afectando potencialmente a la manera en que el lector experimenta el contenido del mismo.

En la lectura clásica cuando un lector abre un libro impreso, se han resuelto ya toda una serie de cuestiones: el libro contiene un texto que se ha decidido leer, disponible en su unicidad e integridad, legible y manejable. Una situación que se presenta como natural para el lector, pues no existe distancia respecto al texto y al medio. Los dispositivos de lectura introducen diferentes grados de distanciamiento, en función de la mayor o menor facilidad de apropiación, en función de la calidad de sus prestaciones, y en función de la permeabilidad e interoperabilidad de sus aplicaciones que, finalmente, determinan diferentes grados de legibilidad. La influencia de las tipografías, los estilos, los diferentes sistemas de codificación espacial y de puesta en página, han sido muy estudiadas en el ámbito impreso, comprobando de qué manera sus formas de presentación influyen en mayor o menor medida en la comprensión de textos. Los estudios pioneros de Richaudeau (1987), Martínez de Sousa (2004) o Herrera Fernandez (1995), y los más recientes de Unger (2009), Buen (2013, 2014), o Mendelsund (2015) constituyen un buen ejemplo de ello. Sin embargo, en el ámbito digital disponemos de pocos estudios para conocer de que manera las diferentes interfaces, las codificaciones tipográficas y espaciales, incrementan o reducen los niveles de legibilidad. Panoz (2013), 
Rodriguez Valero (2016), Eguarás (2014), son algunos de los autores que han tratado un tema crucial en el entorno digital en el que el texto ha de ser adaptable a todo tipo de pantallas y en el que el lector ha de tener la posibilidad de personalizar el sistema gráfico de lectura.

Los tipos de letra constituyen un elemento determinante en la mejora y rapidez de la lectura. Por ejemplo, el diario El País, después de utilizar durante 31 años la letra Times Roman, creada para el diario Times en 1932, y utilizada por casi todos los periódicos del mundo en formato impreso, cambia en 2007 a la letra Majerit, diseñada por el tipógrafo portugués Mario Feliciano, mejor adaptada a las nuevas realidades tecnológicas. El problema es que casi todos los estudios de legibilidad desarrollados en los últimos años, se han centrado en los textos impresos, y se carece aún de referentes reales para cotejar la experiencia de lectura en sistemas cambiantes y tipos de pantalla muy distintos. Esto no quiere decir que no se esté innovando en este terreno. Los diseños de tipografías nuevas no cesan (recientemente Kindle incorporó Bookerly y Google Play Literata a sus aplicaciones de lectura), pero habrán de someterse a estudios en profundidad para comprobar en qué medida responden a necesidades estéticas, ergonómicas, funcionales o económicas.

De cualquier modo, el diseño tipográfico, el desarrollo de aplicaciones y de sus funcionalidades, tiene que ver con lo que Therién (1990) denominó como manipulación, esto es las condiciones materiales del proceso de lectura, estrechamente relacionado con la comprensión y la interpretación. Si en el ámbito impreso la manipulación estaba condicionada por las decisiones del editor, cuyo diseño permanecía inalterable en las manos del lector, en el entorno digital esta tiene que ver con el tipo de dispositivo, el tipo de aplicación, y las funcionalidades incorporadas en las mismas. En el anexo de esta contribución se pueden cotejar las diferencias existentes entre aplicaciones similares, trabajando en entornos diferentes (IOS, Android, dispositivos dedicados). La elección sobre el sistema de lectura determinará los niveles de legibilidad de la misma y las funcionalidades disponibles, diferentes incluso para una misma aplicación en cuando trabaja con sistemas operativos distintos. Además, la legibilidad estará condicionada por factores extrínsecos a las aplicaciones como el tipo de pantalla, o la ergonomía del dispositivo. 
Por lo tanto, al hablar de lectura digital, no se pueden obviar las condiciones en las que se desarrolla la misma, su contexto formal (dispositivo) y propositivo (aplicaciones), pues ello redundará en los otros niveles de la triada, sobre los que, hasta ahora, se han centrado los estudios y la crítica, tanto la especializada como la mediática. Lo que está en juego ahora, con el desarrollo de los contenidos digitales, es el dominio de las formas básicas de manipulación. Esta todavía se encuentra en niveles incipientes que es preciso potenciar como primer paso para facilitar las estrategias de comprensión y los sistemas de interpretación idóneos para cada tipo de texto. Por ello es imprescindible el desarrollo de estudios etnográficos de la información, que permitan el análisis de comportamientos, y la evaluación y diagnóstico del rendimiento de las interfaces existentes, así como el estudio de usabilidad de los diferentes prototipos.

La comprensión, implica la dimensión semiótica del proceso de lectura. Para leer un texto es preciso entender lo que está escrito, lo que implica aspectos lingüísticos, cognitivos y afectivos. Finalmente, la interpretación, se refiere a la dimensión simbólica del proceso de lectura. Leer, en su grado más profundo, es establecer una relación entre el texto que se lee y otros textos que explican, ilustran, completan o amplían lo que se lee. Ahora bien, lo interesante es saber en qué medida la lectura digital favorece o no estos procesos.

\section{La lectura digital y los procesos de comprensión, memorización, interpretación.}

Que la lectura constituye una de esas raras prácticas que concitan la unanimidad en cuanto a su consideración positiva, es algo que nadie pone en duda, incluso en los momentos actuales en los que los discursos de legitimación se han ido diversificando con ponderaciones más distribuidas en torno a otros discursos culturales. La lectura, tomada genéricamente, ha gozado del beneplácito social, al menos desde las postrimerías del siglo XIX, cuando los movimientos en favor de la alfabetización, como medio de integración social, y de promoción y acceso a los bienes culturales pasaron a constituirse en objetivos estratégicos de las políticas estatales. Esto no fue óbice para que existieran movimientos de control respecto a determinados 
tipos de lecturas, motivados por sectores religiosos, políticos, o sociales. Pero incluso estos pronunciamientos, plasmados en diferentes formas de censura, no hacían sino conferirle un estatus especial, aunque fuera por la vía de la negación.

Saber leer se ha considerado como un bien en sí mismo y la incorporación de su aprendizaje a los programas formativos de todos los países del mundo evidencia el grado de consolidación de esta convención. Sin embargo, su evolución y desarrollo está sujeta a múltiples interpretaciones sobre la viabilidad de las estrategias seguidas o sobre la oportunidad de las mismas (Arguelles, 2009).

Las resistencias que todavía abundan, como una especie de reverberación de lo que Gracia (2011) denominaba como el Intelectual Melancólico, se plasman en el fuerte eco de noticias como los efectos negativos de la lectura digital para el sueño, la concentración o la profundidad en la asimilación de contenidos, irán diluyéndose a medida que la investigación vaya demostrando la inconsistencia de análisis basados en criterios metodológicos muy frágiles.

Lo que es una evidencia es que la lectura digital progresa a buen ritmo y amplía sus contextos culturales, sociales e incluso epistemológicos (Van der Weel, 2011), extendiendo sus espacios de influencia. Por lo menos desde la década de 1980 se han venido investigando las circunstancias de la lectura y su relación con lo digital en más de un centenar de estudios. Antes de 1992 la mayoría estos concluían que se leía con más lentitud, con menos precisión y concentración en las pantallas que en el papel (Dillon, A. 1992). Los estudios publicados desde la década de 1990 (Noyes JM; Garland KJ., 2008) sin embargo, han producido resultados más contradictorios: una ligera mayoría ha confirmado las conclusiones anteriores, pero han encontrado pocas diferencias significativas en la velocidad o la comprensión entre el papel y las pantallas de lectura. Y estudios recientes sugieren que, aunque la mayoría de la gente todavía prefiere el papel - especialmente cuando la lectura es intensiva las actitudes están cambiando en la medida en que las formas de presentación y la tecnología de lectura electrónica van mejorando. Sin embargo, experimentos de laboratorio, encuestas e informes sobre el consumo, indican que las pantallas y los dispositivos de lectura no pueden recrear adecuadamente ciertas experiencias táctiles y sensitivas de la lectura en papel 
que, evidentemente, se pierden y, sobre todo, la navegación en textos largos de una manera intuitiva, satisfactoria, y eficaz. A su vez, tales dificultades de navegación pueden inhibir sutilmente la comprensión lectora. En comparación con el papel, las pantallas también pueden demandar una mayor activación de recursos mentales, en detrimento de competencias de carácter cognitivo y memorialístico. Se produce también un problema de actitud. Consciente o inconscientemente, muchas personas se acercan a ordenadores y tabletas con un estado de ánimo menos propicio para el aprendizaje que el que emplean con el papel (Ackerman, R.; Goldsmith, M. 2011).

Algunos estudios recientes muestran poca o ninguna diferencia en cuanto a comprensión entre la lectura en papel o en pantalla (Margolin et al., 2013; Kretszchmar et al., 2013), y otros sugieren que leer textos lineales extensos en una pantalla puede dificultar los procesos subyacentes de comprensión, conocimiento y memoria en un nivel elevado (Ackerman \& Goldsmith, 2011; Jeong, 2012; Kim \& Kim, 2013; Mangen, Walgermo \& Brønnick, 2013;

Comprensión, conocimiento y memoria están estrechamente relacionados. Numerosas investigaciones inciden en la precaria situación de los textos digitales para desencadenar procesos asociativos que faciliten la asimilación y memorización del contenido. El problema podría radicar en la falta de señales físicas o asociaciones que la memoria de una persona puede utilizar para recordar la información. El contexto y los puntos de referencia son importantes para pasar de "recordar" a "saber". Factores aparentemente irrelevantes, como recordar si se lee algo en la parte superior o inferior de la página, si estaba en la página par o impar de un libro o cerca de un gráfico, pueden ayudar a consolidar el recuerdo en la mente.

Esto parece irrelevante en un primer momento, pero el contexto espacial puede ser particularmente importante puesto que, según los psicólogos cognitivos, la evolución puede haber configurado la mente para recordar fácilmente las señales de localización para que podamos encontrar el camino de vuelta. Como señala Morineau (2005), el libro puede ser considerado una representación física de un cuerpo de conocimientos cuya naturaleza es evocada gracias, entre otras cosas, a los elementos paratextuales que comporta (cubierta, tapas, solapas, etcétera). En este sentido, demostró la existencia de una poderosa asociación cognitiva entre la información y su contexto físico a través de un experimento en el que se trabajó con libros convencionales y electrónicos, con el objetivo de verificar las posibilidades de uno y otro 
respecto a la asimilación, la memorización y otras características relacionadas con la comprensión de la información. En general, las prestaciones fueron muy similares, pero señala que la inexistencia de indicadores de memoria, como los arriba señalados, perjudica el efecto de recordatorio y contextualización de la información que en un libro convencional están asegurados.

De especial relevancia para este estudio es la investigación que demuestra los efectos adversos de la intangibilidad espacio temporal de los textos digitalizados en comprensión lectora (Mangen et al., 2013). Cuando los lectores leen en papel tienen un acceso sensorial inmediato a la secuencia del texto, así como a su totalidad; pueden discernir visualmente, así como sentir cinestesicamente su avance página a página a través del texto; el soporte papel proporciona señales físicas, táctiles y espacio-temporales fijas en relación con la extensión del texto (Sellen \& Harper, 2003). Por el contrario, cuando se lee en pantalla, la visión de conjunto de la organización y estructura del texto, la percepción del mismo por parte del lector puede disminuir (Haas, 1996). Aunque esa pérdida de visión de conjunto de la extensión del texto y localización en el mismo puede ser importante para la lectura en general, tener una "percepción del texto" puede ser especialmente importante en el caso de los géneros narrativos. Por un lado, porque están basados en una sucesión de acciones y sucesos cronológicamente ordenados, y un sentido cinestésico paralelo del desarrollo de la actividad lectora puede mantener la inmersión en el mundo de la narración; por otro lado, la separación de ese sentido de la percepción física de la actividad lectora puede ser precisamente lo que impida la inmersión en el mundo de la narración, o causar la sensación de pérdida en el mismo.

Anne Mangen, desarrolló una investigación (Mangen, A.; Walgermo, B.R.; Bronnick, K.2013) en la que pidieron a 72 estudiantes con capacidad de lectura similar leer dos tipos de textos, una de carácter ensayístico y otro de narrativa, cada uno de aproximadamente 1500 palabras de extensión. La mitad de los alumnos leyeron los textos en papel y la otra mitad en digital, mediante archivos PDF, en ordenadores con monitores de pantalla de cristal líquido de 15 pulgadas (LCD). Posteriormente se sometió a los alumnos a pruebas de comprensión con preguntas de opción múltiple y respuestas cortas. Los resultados de los estudiantes que leyeron en digital fueron peores que los de 
los estudiantes que lo hicieron en papel. Mangen cree que los estudiantes que leyeron PDF encuentran más dificultades de búsqueda y localización de las informaciones. En el ordenador solo podían desplazarse a través de los archivos o secciones del PDF, mientras que los estudiantes que leyeron en papel podían sostener el texto en su totalidad en sus manos y cambiar rápidamente entre diferentes páginas. Debido a su fácil navegabilidad, los libros de papel y documentos pueden ser más adecuados para la asimilación de un texto. Según Mangen, la posibilidad de despreocuparse de los sistemas de localización, gracias a los mapas cognitivos que elabora el lector cuando se encuentra ante una obra impresa, libera capacidad cognitiva y de comprensión hacia el contenido. Son abundantes los estudios que indican que las pantallas y dispositivos de lectura electrónica todavía interfieren dos aspectos importantes relacionados con la buscabilidad y navegación: la serendipia y el control y sentido de la personalización sobre lo leído. Subyayar, anotar, resaltar con tinta sobre las obras, reforzaría el carácter de recordatorio que estas entrañan (Gerlach, J.; Buxman, P. 2011); y únicamente en la medida en que esas favorezcan los procesos de socialización se compensaran las desventajas que la homogeneidad formal puede representar. La pérdida de estos elementos contextuales es inevitable en un dispositivo en el que se pueden almacenar cientos de libros, pero en el que únicamente se contempla la página que se está leyendo en ese momento y lo leído y por leer es una simple indicación numérica o porcentual.

El problema radica, en gran medida, en la falta de percepción global del espacio informativo en el que se encuentra el lector, de tal manera que al desplazarse por el corpus digital puede carecer de información concreta acerca del volumen y organización de los contenidos puestos a su disposición. Un libro cualquiera, una enciclopedia tradicional, una estantería de una biblioteca indican, por su mera presencia física, la cantidad de información que presumiblemente contienen. En el caso de una obra electrónica esta dimensión es puramente abstracta. Para que la navegación pueda efectuarse sin contratiempos es precisa la existencia de directivas espaciales que permitan moverse con fluidez en el espacio informativo sin perder el rumbo. El lector ha de interiorizar la estructura del espacio informativo en que se encuentra, en definitiva, los modos operativos del programa, de la aplicación, que en la medida de lo posible coincidirán con los modos operativos de la mente. El modelo de lectura tradicional se ve considerablemente alterado al faltar la 
representación mental de la estructura del documento, que por sí misma es generadora de sentido. El lector familiarizado con los documentos tradicionales desarrolla toda una serie de hipótesis perceptivas a la hora de consultarlos prediciendo, en cierto modo, el lugar aproximado en el que puede encontrar la información, valiéndose para ellos de un modelo de representación estructural asimilado de manera inconsciente a través del contacto habitual con estos medios. Con los documentos electrónicos esta verificación se complica dada la ausencia física de la información disponible, cuya cantidad en ningún momento puede adivinar, lo cual contribuye a dificultar este factor de reconocimiento orientativo.

El otro gran problema es el de la atención, relacionado con la aparición de elementos distractivos y la consiguiente falta de concentración. Julie Bosman y Matt Richel (2012) sostienen que el libro electrónico se sitúa en el centro de una red diseñada para desviar la atención. La lectura en los teléfonos inteligentes conectados, las tabletas, Internet a un clic de distancia, siempre esperando debajo de la superficie de la página, representan un elemento de permanente distracción para el lector.

En el entorno de la lectura digital son numerosos los focos de atención, lo que la hacen poco propicia para la profundidad, multiplicándose las ocasiones que favorecen la desconcentración. Sin embargo, más que un estilo cognitivo generacional se trataría de una característica propia de los documentos digitales, en tanto que se trata de un medio que precisa de la capacidad de articular diferentes velocidades y modos de lectura, así como la de reducir la sobrecarga operatoria previa a la lectura sostenida. Se habla de sobrecarga cognitiva cuando los individuos, en el marco de una operación principal, en este caso la lectura, se encuentran con diferentes situaciones, y necesitan tomar decisiones que dependen de otras operaciones tan numerosas que ocultan o dificultan la tarea principal. Por lo tanto, esta sobrecarga es operacional, está ligada a la atención y se diferencia de la sobrecarga informativa.

Pero la lectura digital y el grado de profundidad desarrollado en la misma, puede estar también vinculado con elementos de carácter emocional o de suposiciones vinculadas con el pacto de lectura que se desarrolla en función de determinados tipos de textos. Una interesante investigación desarrollada 
por Mangen (2014b) muestra como la superficialidad y eficacia en las prácticas de lectura puede estar condicionada por la información sobre el género que, presumiblemente, se está leyendo. Un mismo texto, demuestra esta investigadora, no se lee de la misma manera si se piensa que es una narración literaria, que si se cree que es un artículo periodístico.

Son muchas las obras que se publican alertando de los peligros de la perdida de profundidad y de las distracciones. Cunningham (2014), Biagini (2015) Ulin (2010), Baron (2015) y sobre todo Carr (2011a, 2011b). El éxito de Carr radica en haber extendido las interrogantes sobre las dificultades de concentración al campo de la lectura digital, llegando a la conclusión de que "no se piensa de la misma manera que antes". Lo que Carr y otros estudiosos como Maryanne Wolf (2010) constatan es que la práctica de la lectura digital, como práctica cultural, está lejos de cumplir las especificaciones de la lectura heredada de la lectura clásica, sobre todo las relativas al triángulo lectura / memoria / pensamiento. Ahora bien, una de las debilidades de Carr y otros pensadores en esta línea radica en la subestimación de la implicación del lector y del papel de la formación, imprescindible en la articulación de prácticas regladas en igualdad de condiciones que las que en su momento articularon la alfabetización tradicional. La educación convencional, todavía moviliza una atención principalmente orientada hacia el texto, mientras que la cultura digital se corresponde más con una atención orientada hacia el medio. La atención orientada hacia el texto, se fundamenta y favorece por el libro que, precisamente como medio tiende a desaparecer en beneficio del texto (Giffard, 2013). La forma tipográfica del libro influye poderosamente sobre la recepción del lector. En este contexto el lector tiende a concentrarse sobre el texto en detrimento de cualquier otra forma de actividad, desarrollada como rutina. Sin embargo, los formatos digitales no dejan olvidar el medio tan fácilmente. La atención se orienta hacia el medio y su funcionamiento es determinante en el proceso de comunicación, de tal manera que juega un papel predominante en la lectura digital. Los conflictos de atención se originan generalmente entre las interferencias que se pueden dar entre texto y medio, $y$ son característicos de las generaciones más jóvenes propensas a fijarse en las múltiples posibilidades del medio en detrimento del texto. 


\section{A modo de conclusión}

La historia, breve, de la lectura digital se ha ido adaptando a dos modelos que, en cierto modo, integran la evolución tecnológica y epistemológica de la lectura en la corriente general de comportamiento de las innovaciones tecnológicas, en la medida en la que una de las sendas reviste un carácter imitativo con respecto al impreso, y la otra una pretensión de clara diferenciación con respecto a este. Se podría dividir la historia de la lectura digital entre el periodo previo a la web y el posterior. Sin embargo, lo que realmente constituye un eslabón determinante en el desarrollo de la lectura digital ha sido la aparición de los dispositivos de lectura especializados, del Ipad y demás tabletas y de dispositivos de lectura móvil. La década transcurrida desde entonces representa el núcleo principal en el que se concentran los principales hitos relacionados con la lectura digital. A lo largo de la misma hemos visto desarrollarse dos circuitos concurrentes pero diferenciados. Uno de carácter conservador, imitativo de lo impreso, encarnado en los dispositivos de tinta electrónica, con destacados ejemplos en legibilidad y prestaciones como el Kindle, Sony o Kobo, y otro de carácter polivalente y multitarea representado por las tablets y smartphones, en los que aunque la lectura no constituye su función principal, los desarrollos de aplicaciones y de funcionalidades multimedia le confieren unas posibilidades de desarrollo editorial completamente novedosas y abiertas a la experimentación permanente. No son dos circuitos excluyentes, pues el "skeuomorphism", esto es la práctica de incorporar elementos obsoletos en un diseño, por familiaridad por tradición, a pesar de que ya no sirven a ningún propósito funcional, sigue siendo muy potente en el ámbito del libro digital (Sinclair; Rockwell, 2014)

Libros y lectura han guardado una curiosa relación a lo largo de la historia y, singularmente, desde la invención de la imprenta: mientras los libros han ido creciendo en progresión geométrica la lectura lo ha hecho en progresión aritmética, describiendo una relación que en muchos casos se ha convertido, paradójicamente, en inversamente proporcional, generando una de las grandes contradicciones de nuestro tiempo. El crecimiento exponencial de la publicación de obras, no ha ido acompañado de un movimiento similar en la lectura de las mismas. Si la lectura y el pensamiento crítico que acompaña a la misma constituye uno de los signos de la modernidad, la retracción que, 
aparentemente, está experimentando ésta representaría otro de los síntomas de la desintegración de una fenomenología bibliocentrica fagocitada por la aparición de medios de expresión más eficaces desde el punto de vista comunicativo.

Además, el ecosistema tradicional del libro y de la lectura se ha visto alterado por la aparición de tecnologías de carácter disruptivo que, por primera vez en la historia de la edición, afectan a toda la cadena de valor del libro, desde la autoría a la recepción, articulando un sistema nuevo, emergente, que ya está produciendo desplazamientos significativos, cuando no migraciones irreversibles, entre los soportes impresos y digitales. Las tensiones y conflictos derivados de esta dialéctica se han sustanciado de diferentes modos, generando movimiento de resistencia, bien por la vía del rechazo, bien por la de la asimilación.

Las cifras que arrojan los diferentes estudios e informes que se publican en todo el mundo representan un elevado grado de inconcreción, por lo que es difícil saber cuánto se lee y, sobre todo, cómo se lee. Todos los países han incorporado la cuantificación de lo digital en sus anuarios, panorámicas y observatorios, pero las cifras que arrojan revisten un carácter siempre aproximativo, pues la irrupción de la autopublicación como uno de los escenarios más potentes del ecosistema digital dificulta la normalización de la práctica estadística en este terreno. De tal manera que la industria editorial de cada país, o los organismos encargados de su estudio, dan fe de las prácticas de publicación que podíamos considerar como "regladas", regidas por estándares internacionales y sujetas a sistemas de control bibliográfico que garantizan la fiabilidad de las cifras, pero queda un extenso margen, cada vez menos periférico, que es preciso tener presente cuando se contempla esta nueva realidad.

Esto no quiere decir que se haya abandonado la fase de transición en la que todavía nos encontramos, con modelos incipientes cuya consolidación requerirá todavía años de maduración del mercado, pero se van poniendo las mimbres de un sistema que será predominante a medio plazo, por motivos no sólo económicos, sino sociológicos y culturales.

Finalmente, la clave del desarrollo de la lectura digital radica en la formación. La construcción del lector digital exige la adquisición de un conjunto de habilidades y competencias que le faculten para una adecuada apropiación de 
los dispositivos y de los contextos tecnológicos que facilitan las diferentes formas de legibilidad. Gran parte de la debilidad e inconsistencia de muchas de las investigaciones desarrolladas radica, precisamente, en no haber comprendido que no se pueden comparar realidades cualitativamente diferentes, y menos aún derivar de ellas conclusiones significativas. Cuando se comparan prácticas de lectura en términos de comprensión, memorización, etc. se hace con grupos de individuos educados en la lectura impresa, sin formación sólida en la lectura digital, por lo que los resultados, no pueden ser más que meramente orientadores. El futuro de la investigación en el ámbito de la lectura digital, pasa irremediablemente por la inserción de esta en la educación, como una práctica regladas más.

\section{Referencias}

ACKERMAN, R. and M. GOLDSMITH, 2011. Metacognitive regulation of text learning: on screen versus on paper. Journal of Experimental Psychology: Applied, 17(1), 18-32. eISSN 1939-2192.

ARGUELLES, Juan Domingo, 2009. Si quiere lee: contra la obligación de leer y otras utopias lectoras. Madrid: Fórcola. ISBN 978-84-936321-1-3.

BARON, N. S., 2015. Words Onscreen: The fate of reading in a Digital World. New York: Oxford University Press. ISBN 978-0-19931576-5

BENHAMOU, Françoise, 2014. Le livre à l'heure numérique: papier, écran, vers un nowveau vagabondage. Paris, Seuil. ISBN 978-2-02114060-6

BLEICH, David, 1978. Subjective Criticism. Baltimore: John Hopkins University Press.

BOSSMAN, Julie and Matt RICHEL, 2012. Finding your book interrupted ... by the tablet you read it on. The New York Times. Media and Advertising. [Consulta: 15-112016]. Disponible en: http://www.nytimes.com/2012/03/05/business/media/ebooks-on-tablets-fight-digital-distractions.html?pagewanted=all

BOURDIEU, Pierre, 2011. Las reglas del arte: génesis y estructura de campo literario. Barcelona: Anagrama. ISBN 978-84-339-1397-5. 
BOURDIEU, Pierre y Roger CHARTIER, 2011. El sociólogo y el historiador. Madrid: Abada. ISBN: 978-84-15289-22-7.

BOURDIEU Pierre, 2012. La distinción: criterios y bases sociales del gusto. Madrid, Taurus. ISBN: 978-84-306-0911-6.

BUEN Unna, Jorge de, 2013. Diseño, comunicación y neurociencias. Gijón, Trea. ISBN 978-84-9704-741-8.

BUEN Unna, Jorge de, 2014. Manual de diseño editorial. Gijón, Trea ISBN 978-849704-762-3.

CARR, N., 2011a. Superficiales: ¿qué está haciendo Internet con nuestras mentes?. Madrid: Taurus.

CARR, N., 2011b. ¿Google nos hace estúpidos? Madrid: Taurus.

CHARTIER, Roger, 2000. Las revoluciones de la cultura escrita. Barcelona: Gedisa. ISBN: 978-84-7432-829-5

CUNNINGHAM, A.E., 2014. Book smart: How to develop and support successful, motivated readers. New York: Oxford University Press.

DARNTON, Robert, 2010. Las razones del libro: futuro, presente, pasado. Madrid, Trama.

ECO, Umberto, 1999. Lector in Fabula. Barcelona: Lumen. ISBN: 978-84-264-11426.

EGUARÁS, Mariana, 2014. Tipografías para libros impresos y digitales. Consultoría Editorial Mariana Eguarás.

EMERSON, Lori, 2014. Reading writing interfaces: from the digital to the bookbound. Minneapolis. Minneapolis: University of Minnesota Press. ISBN 978-0-8166- 9125-8.

FISH, Stanley, 1990. Is There a text in this class? The authority of interpretive communities. Cambridge (USA): Harvard University Press, ISBN 978-0-67446726-2.

FURTADO, José Afonso. 2007. El papel y el pixel: de lo impreso a lo digital: continuidades y transformaciones. Gijón: Trea. ISBN: 978-84-9704-300-7. 
GERLACH, J., and P. BUXMANN, P., 2011. Investigating the acceptance of electronic books: The impact of haptic dissonance on innovation adoption. European Conference on Information Systems (ECIS).

GIFFARD, A., 2013. Rhétorique de l'attention et de la lecture. Entretiens du Nouveau Monde Industriel, 14-44.

GÓMEZ DIAZ, Raquel, et al. 2016. Leyendo entre pantallas. Gijón, Trea. ISBN 97884-9704-945-0

GRACIA, Jordi, 2011. El intelectual melancólico. Barcelona, Anagrama.

HAAS, C., 1996. Writing technology: studies on the materiality of literacy. Mahwah, NJ: L. Erlbaum Associates.

HERRERA FERNÁNDEZ, Eduardo, 1995 La presentación visual del lenguaje: conexiones entre forma y legibilidad. Vitoria-Gasteiz: Universidad del País Vasco. ISBN 978-84-7585-683-4

HOUSTON, Keith, 2016. The Book: a cover-to-cover exploration of the most powerful object of our time. W. W. Norton \& Company. ISBN 978-0-39324479-3.

ISER, Wolfgang, 1987. El acto de leer. Madrid: Taurus. ISBN 978-84-306-2176-7.

ISER, Wolfgang, 2005. Rutas de la interpretación. México: Fondo de Cultura Económica

ISBN 978-968-167327-7.

JAUSS, Hans Robert, 2012. Caminos de la comprensión. Madrid: Machado Libros. ISBN 978-84-7774-941-7.

JEONG, H., 2012. A comparison of the influence of electronic books and paper books on reading comprehension, eye fatigue, and perception. The Electronic Library, 30, 390-408. DOI: 10.1108/02640471211241663

KRETZSCHMAR, F., et al., 2013. Subjective impressions do not mirror online reading effort: concurrent EEG-eye tracking evidence from the reading of books and

digital media. PloS one, 8(2), e56178. DOI: 10.1371/journal.pone.0056178 
MANGEN, A., B.R. WALGERMO and K. BRONNICK, K., 2013. Reading linear texts on paper versus computer screen: Effects on reading comprehension. International Journal of Educational Research, 58, 61-68.

MANGEN, A. et. al. (2014a). Mystery story reading in pocket print book and on kindle: Possible impact on chronological events memory. Conference paper presentation, IGEL (The International Society for the Empirical Study of Literature and Media), Turin, Italy July 21-25.

MANGEN, A., \& KUIKEN, D. (2014b). Lost in an iPad: narrative engagement on paper and tablet. Scientific Study of Literature, 4(2), 150-177.

MANGEN, A., and A. van der Weel, A., 2016. The evolution of reading in the age of digitisation: an integrative framework for reading research. Literacy, 50(3): 116124. doi: $10.1111 /$ lit.12086.

MARGOLIN, S. J., et al., 2013. E-readers, computer screens, or paper: Does reading comprehension change across media platforms? Applied Cognitive Psychology, 27, 512-519. DOI: $10.1002 /$ acp.2930.

MARTINEZ DE SOUSA, José, 2004. Manual de edición y autoedición. Madrid, Pirámide. ISBN 978-84-368-0840-7.

MENDELSUND, Peter, 2015. Qué vemos cuando leemos. Barcelona, Seix Barral. ISBN: 978-84-322-2519-2.

MORINEAU, et al., 2005. The emergence of the contextual role of the e-book in cognitive processes through an ecological and functional analysis. International Journal of Human-Computer Studies, 62, 329-348.

NOYES JM, and KJ GARLAND, 2008. Computer-vs.paper-based tasks: are they equivalent? Ergonomics, 51 (9):1352-75.

PANOZ, Jiminy, 2013. Design du livre numérique. Paris, Walrus.

RICHAUDEAU, Francois et al., 1987. La legibilidad: investigaciones actuales. Madrid, Fundación Germán Sánchez Ruipérez. ISBN 978-84-368-0358-7.

RODRÍGUEZ-VALERO, Daniel, 2016. Manual de tipografía digital. Valencia: Campgràfic. ISBN 978-84-96657-46-5. 
SELLEN, A. J., and R.H.Harper, 2003. The myth of the paperless office. Cambridge: The MIT Press.

SINCLAIR, S. and G.ROCKWELL, G., 2014. Les potentialités du texte numérique. Dans: M.E. SINATRA and M. VITALI-ROSATI, eds, Pratiques de l'édition numérique Montreal: Presses de l’Université de Montréal, 191-204.

THERIEN, Gilles, 1990. Pour une sémiotique de la lectura. Protée, 18(2), 67-80.

ULIN, D.L., 2010. The lost of Art of Reading: why books matter in a distracted age. Seattle: Sasquatch Books.

UNGER, Gerard, 2009. ¿Qué ocurre mientras lees? tipografía y legibilidad. Valencia, Campgràfic. ISBN 978-84-96657-12-0.

VAN DER WEEL, Adriaan, 2011. Changing our textual minds: towards a digital order of knowledge. Manchester: Manchester University Press. [Consulta: 15-11-2016]. Disponible en: http://www.let.leidenuniv.nl/wgbw/research/Weel_Articles/Weel_Changing_MU P.pdf

VANDERDORPE, C., 2011. Some considerations about the future of reading. Digital Studies / Le Champ Numérique, 2(2).

WOLF, M., 2010. Cómo aprendemos a leer. Barcelona: Ediciones B. 